\title{
Ultra-high Cleanliness of ISO Class Minus 2 Realized by Clean-Unit System Platform for Integrating the Bottom-up and Top-down Systems
}

\author{
Md. Dalilur Rahaman ${ }^{{ }^{1}}$, Hideo Kaiju ${ }^{2}$ and Akira Ishibashi ${ }^{2}$ \\ ${ }^{1}$ Department of Physics, Dhaka University, Dhaka-1000, Bangladesh \\ ${ }^{2}$ Laboratory of Quantum Electronics, Research Institute for Electronic Science \\ Hokkaido University, Sapporo 060-0812, Japan
}

(Received : 13 June 2012; Accepted : 22 November 2012)

\begin{abstract}
The time dependence of the airborne particle count and the cleanliness of an airtight stainless steel clean-unit system platform (S-CUSP) with $100 \%$ air feedback through the feedback loop by installing the ultra low penetration air (ULPA) filter just beneath the high efficiency particulate air (HEPA) filter in the feedback loop has been studied. By controlling the number of particles coming out from the HEPA filter, the ultra high cleanliness of ISO class minus 2 has been obtained, which is five orders of magnitude better than that of the super cleanroom (ISO Class 3). Analyses of the experimental results demonstrate that the S-CUSP with flat feedback loop would be a viable economical means to achieve the more stringent cleanliness class, which has the immense importance for expediting the multi-disciplinary experiments and production fields such as nanotechnologies and biotechnologies.
\end{abstract}

Keywords: Ultra-high cleanliness, Nanotechnology, Biotechnology, Top-down and Bottom-up Systems and Crossdisciplinary Research

\section{Introduction}

The requirement of the contamination control is a crucial factor in many high-technology manufacturing, such as nanotechnology and biotechnology, because the maximum size of particle which is tolerable is one tenth of the dimension of the critical feature ${ }^{1-4}$. On the contrary, the cost-effectiveness is the vital issue for the ultra-high cleanliness environment for nanotechnology, biotechnology and food industries. 5 . In recent years, mini-environments have been developed in cost-effective ways but their cleanliness is not promising ${ }^{6-8}$. The particle sizes which were adversely effective to previous generation of semiconductor devices are now being the "killer" particles for the nano-scale device fabrication. On the other hand, the food industry is also striving to improve the shelf life and decrease food poisoning incidences to satisfy the demand of consumers for natural food products ${ }^{5}$. So, it is necessary to fabricate a particulate-contamination-free production or manufacturing environment for optimizing the product yield.

Integration of a bottom-up system with a top-down one through the fusion of tool-based and device-based approaches has already been proposed which is an important issue in the field of nanotechnology ${ }^{9}$. A clean-unit system platform (CUSP), made of multiply connectable airtight clean box unit with $100 \%$ air feedback through a feedback loop, is the tool-based approach ${ }^{10}$. Cleanliness of ISO class 1 2 has already been demonstrated in airtight acrylic based CUSP with single high efficiency particulate air (HEPA) filter $^{11}$. On the other hand, ultra-high cleanliness of ISO class minus 1 has been realized in airtight acrylic based CUSP with the conjunction of the HEPA filter just beneath the ultra low penetration air (ULPA) filter and isolation of the particle counter from the CUSP interior volume ${ }^{12}$, whereas a super cleanroom has the cleanliness of ISO class $3^{13}$. In the acrylic based CUSP, the feedback loop has a bent shape. As a consequence, it requires extra area for installing in the existing facilities, whereas the stainless steel cleanunit system platform (S-CUSP) with flat feedback loop does not. Acrylic based CUSP and the S-CUSP can effectively isolate the product from the major contamination source, which is one of the key requirements for optimizing the product yield.

In the present study, we investigate the time dependence of the airborne particle count in the airtight S-CUSP with 100 $\%$ air feedback through flat feedback loop with the conjunction of the double filters in order to ascertain the more stringent degree of cleanliness.

\section{Experimental Methods}

Figures 1(a) and 1(b) show the schematic illustration of the acrylic based CUSP with the single HEPA filter and double filters, respectively. The S-CUSP with the single HEPA filter and the double filters is depicted in Fig. 1(c) and 1(d).

The unit of CUSP is a multiply-connectable clean box that consists of the main chamber, the HEPA filter, a set of gloves, a feedback loop, and connection boxes at the sides and back. The chamber of the CUSP unit has a $0.45 \times 0.8 \mathrm{~m}^{2}$ rectangular top, a $0.55 \times 0.8 \mathrm{~m}^{2}$ rectangular bottom, and trapezoidal sides having $0.45-\mathrm{m}$-long top sides, $0.55-\mathrm{m}$-long bottom sides, and heights of $0.8 \mathrm{~m}^{11}$. The particle reduction rate of the HEPA and ULPA filters for the particle diameter of $0.3 \mu \mathrm{m}$ and $0.15 \mu \mathrm{m}$ is $99.997 \%$ and $99.99999 \%$, respectively. The number of airborne particles was measured in the acrylic based CUSP and the S-CUSP by the particle counters, LASAIR 110 and LASAIR 310, made by PMS Corp. at room temperature.

These particle counters can measure the number of airborne particle of diameter of $0.1,0.2,0.3$, and $0.5 \mu \mathrm{m}$, and 0.3 , $0.7,1.0,5.0$, and $10.0 \mu \mathrm{m}$ per cubic foot per minute, respectively. For performing the longer period measurement of the number of airborne particle in the S-CUSP with the conjunction of the ULPA filter just beneath the HEPA filter,

\footnotetext{
*11Author for Correspondence,e-mail: dalilurrahaman1976@gmail.com
} 
we set the particle counting and waiting time as $10 \mathrm{~min}$ and $10 \mathrm{~s}$, respectively.

Theoretically, the airborne particle density, $n(t)$, in the airtight acrylic based CUSP and the S-CUSP with the single
HEPA filter and the $100 \%$ air feedback through the feedback loop is given by a solution of the differential equation as ${ }^{10}$

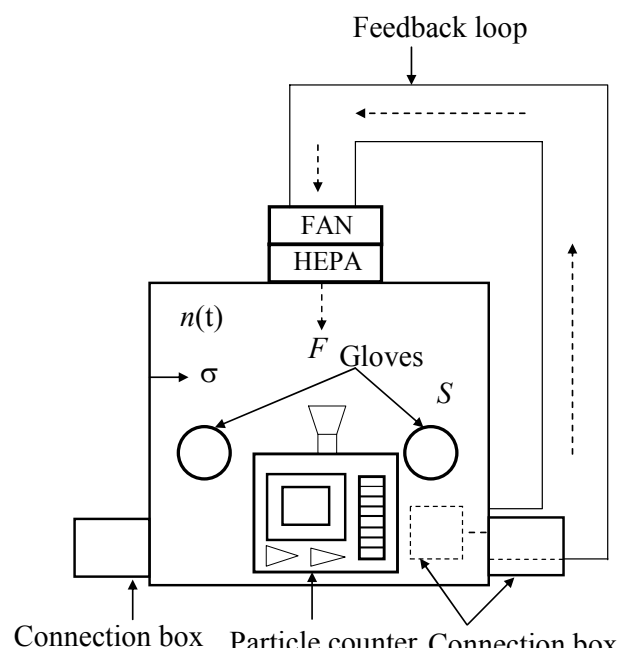

(a)

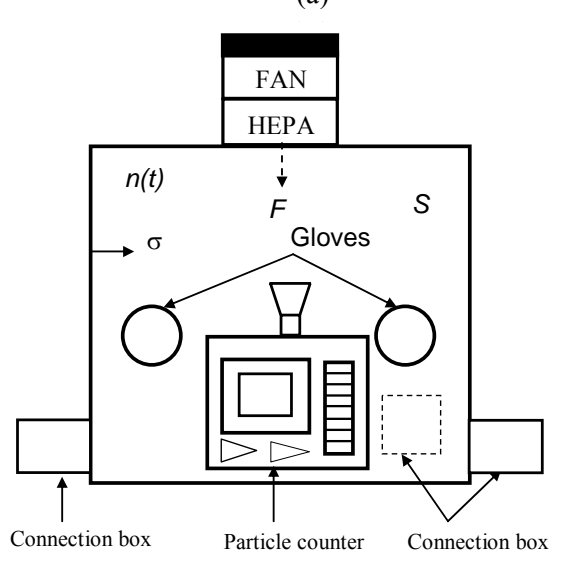

(c)

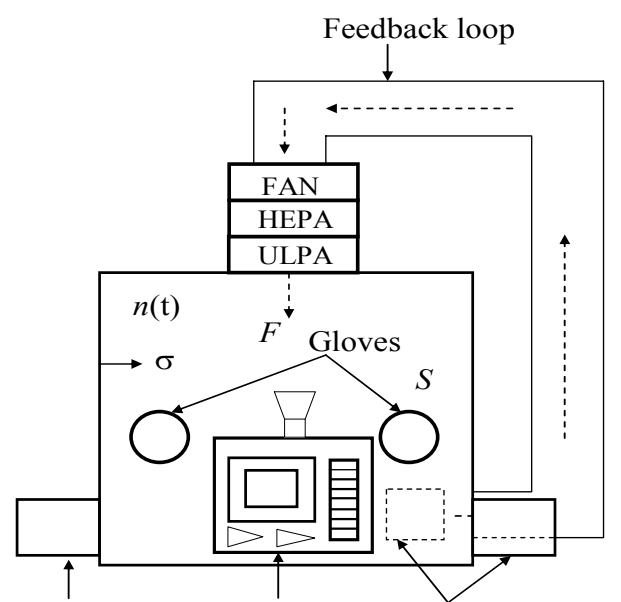

Connection box Particle counter Connection box

(b)

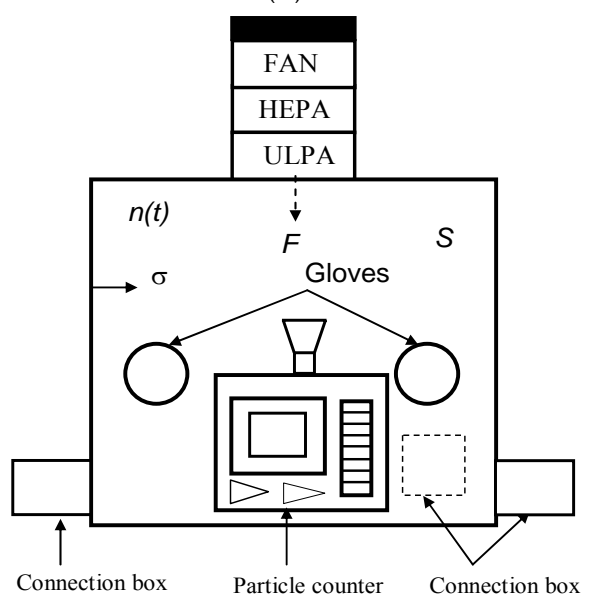

(d)

Fig. 1. Schematic illustration of the acrylic based CUSP with (a) the single HEPA filter and (b) the double filters and S-CUSP with (c) the single HEPA filter and (d) the double filters.

$$
\begin{aligned}
& V \frac{d n(t)}{d t}=S \sigma-n(t) F+n(t) F(1-\gamma) \\
& =S \sigma-n(t) F \gamma
\end{aligned}
$$

where $V$ is the volume of the chamber, $S$ is the area of the inner surface of the clean unit, $\sigma$ is the number of particles emitted from the wall of acrylic based CUSP and the S-CUSP unit per unit time per unit area, $F$ is the flow rate, and $\gamma$ is a reduction rate of particles by the HEPA filter. The solution of eq. (1) under the boundary condition, $n(0)=N_{0}$, is given by ${ }^{10}$

$$
n(t)=\frac{S \sigma}{\gamma F}+\left(N_{0}-\frac{S \sigma}{\gamma F}\right) \exp \left(-\frac{\gamma F}{V}\right) t
$$

where $N_{0}$ is the ambient dust density.

At $t \rightarrow \infty$, the steady state airborne particle density in the acrylic based CUSP and the S-CUSP unit with the single HEPA filter is

$$
n(\infty)=\frac{S \sigma}{\gamma F}
$$

The time dependence of the airborne particle count in the acrylic based CUSP and the S-CUSP with single HEPA filter is investigated using eqs. (2) and (3).

Theoretically, the airborne particle density, $n(t)$, in the acrylic based CUSP and the S-CUSP with the conjunction of the HEPA and ULPA filters and $100 \%$ air feedback through the feedback loop is obtained by a solution of the differential equation as ${ }^{12}$ 


$$
\begin{aligned}
& V \frac{d n(t)}{d t}=S \sigma-n(t) F+n(t) F\left(1-\gamma_{1}\right)\left(1-\gamma_{2}\right) \\
& =S \sigma-n(t) F\left(\gamma_{1}+\gamma_{2}-\gamma_{1} \gamma_{2}\right)
\end{aligned}
$$

where $\gamma_{1}$ and $\gamma_{2}$ are the particle arrest efficiency of the HEPA and ULPA filters, respectively. The solution of eq. (4) under the boundary condition $n(0)=N_{0}$, is obtained as ${ }^{12}$

$$
\begin{aligned}
& n(t)=\frac{S \sigma}{F\left(\gamma_{1}+\gamma_{2}-\gamma_{1} \gamma_{2}\right)}+ \\
& \left(N_{0}-\frac{S \sigma}{F\left(\gamma_{1}+\gamma_{2}-\gamma_{1} \gamma_{2}\right)}\right) \exp \left(-\frac{F\left(\gamma_{1}+\gamma_{2}-\gamma_{1} \gamma_{2}\right)}{V}\right) t
\end{aligned}
$$

At $t \rightarrow \infty$, the steady state airborne particle density in the acrylic based CUSP and the S-CUSP with the combination of HEPA and ULPA filters is

$$
n(\infty)=\frac{S \sigma}{F\left(\gamma_{1}+\gamma_{2}-\gamma_{1} \gamma_{2}\right)}
$$

For simplification, we have assumed that $1-\gamma_{1}=\delta$ and $1-\gamma_{2}=\varepsilon$, where $1>>\delta>>\varepsilon$. Therefore, the denominator of eq. (6) becomes $F$ $\left[\gamma_{1}\left(1-\gamma_{2}\right)+\gamma_{2}\right]=F\left(\gamma_{1} \varepsilon+\gamma_{2}\right) \rightarrow \gamma_{2} F$ as $\gamma_{1} \varepsilon \rightarrow 0$.

So, we can write eq. (5) as

$$
n(t)=\frac{S \sigma}{\gamma_{2} F}+\left(N_{0}-\frac{S \sigma}{\gamma_{2} F}\right) \exp \left(-\frac{\gamma_{2} F}{V}\right) t,
$$

At time $t \rightarrow \infty$, the steady state airborne particle density in the acrylic based CUSP and the S-CUSP unit with the double filters is

$$
n(\infty)=\frac{S \sigma}{\gamma_{2} F}
$$

The time dependence of airborne particle count in the acrylic based CUSP and the S-CUSP unit with the combination of HEPA and ULPA filters is investigated using eqs. (7) and (8).

\section{Results and Discussion}

Figure 2(a) shows the time dependence of the airborne particle count in the S-CUSP, with the combination of the double filters (HEPA and ULPA) in the feedback loop, for the particle diameter of $0.3 \mu \mathrm{m}$. When the fan filter unit is switched on, at $t=0$, the airborne particle count is the same as the ambient particle density. The measurement of the time dependence of the airborne particle count in the S-CUSP is performed for 58 days based on the counting time of $10 \mathrm{~min}$. and waiting time of $10 \mathrm{~s}$. The inset shows the time dependence of airborne particle count in the early stage after the S-CUSP is switched on, representing the particle count decay behavior. The solid circles with error bars and the solid line represent the experimental and the theoretical results, respectively. The particle count goes to zero within 10 minutes. The particle count decay time is given by $\tau_{\mathrm{c}}=V / \gamma_{2} F^{10}$. By using the volume $V$ of $0.32 \mathrm{~m}^{3}$, the flow rate $F$ of $0.92 \mathrm{~m}^{3} \mathrm{~min}^{-1}$ and the particle arrest efficiency of the ULPA filter $\gamma_{2}$ of 0.9999999 in the aforementioned relation, theoretically we obtained the particle count decay time, $\tau_{c}$, which was $20 \mathrm{~s}$.

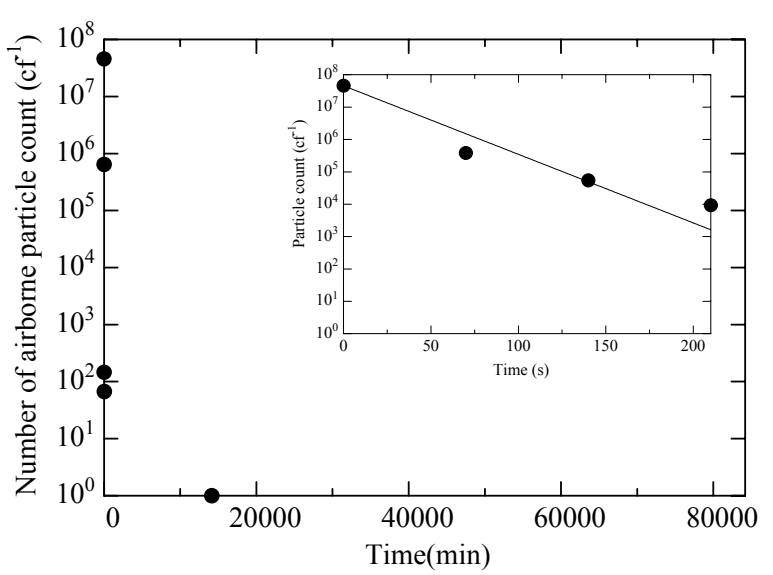

Fig. 2(a). Time dependence of the airborne particle count in the SCUSP with double filters.

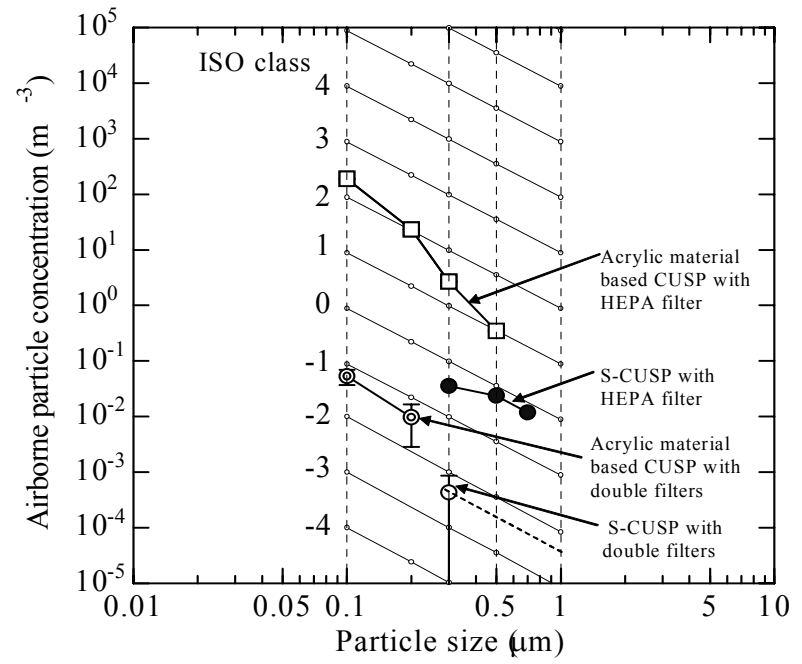

Fig.2 (b). Cleanliness of S-CUSP with double filters.

By fitting the experimental results, the obtained particle count decay time, $\tau_{c}$, is $20.5 \mathrm{~s}$. The theoretical and the experimental results of the particle count decay time are of the same order of the magnitude.

Figure 2(b) shows the cleanliness of the acrylic based CUSP and S-CUSP with the single HEPA filter as well as the double filters (HEPA \& ULPA). The open squares and the double circles with error bars represent the cleanliness of the acrylic material based CUSP with the single HEPA filter and the double filters, respectively. The cleanliness of ISO Class 1 2 in the acrylic based CUSP with the single HEPA filter has been obtained with the reduction of the number of particles coming out from the interior wall of the acrylic based CUSP ${ }^{11}$. When the cleanliness of ISO class 1 2 has been obtained, the number of airborne particles coming out from the inner surface per unit area per unit time is 0.83 $\mathrm{m}^{-2} \mathrm{~s}^{-111}$. 
On the other hand, the cleanliness of ISO class minus 1 has been obtained in the acrylic based CUSP by controlling the number of airborne particles coming out from the HEPA filter with the conjunction of ULPA filter just beneath the HEPA filter and the isolation of the particle counter from the CUSP interior volume ${ }^{12}$. The solid circles represent the cleanliness of the S-CUSP with the single HEPA filter which lies between the ISO Class $0 \sim$ minus 1 . The reason for obtaining this result is that the number of physically adsorbed particles coming out from the inner wall surface of S-CUSP is small. The open circle with the error bar represents the cleanliness of the S-CUSP with the double filters and the dashed line represents the theoretical prediction.

This result is attributed due to the installation of the ULPA filter just beneath the HEPA filter, which means that by controlling the particles coming out from the HEPA filter as well as the physically adsorbed particles coming out from the interior surface of the S-CUSP is $2.3 \times 10^{-6} \mathrm{~m}^{-2} \mathrm{~s}^{-1}$. Another important point is needed to be considered for obtaining the ultra-high cleanliness of ISO Class minus 2, which is the perfect air filtration through the filter of the LASAIR 310 particle counter. Thanks to multiple connectability of the clean unit, S-CUSP has the potential application as tool of realizing the ultra-high cleanliness for cross-disciplinary research.

\section{Conclusion}

Cleanliness of the S-CUSP with the flat feedback loop has been studied in order to achieve the ultra-high clean environment and the time dependence of the airborne particle count is clarified theoretically and experimentally. We have succeeded in realizing the ultra-high cleanliness of ISO class minus 2 in the S-CUSP with the flat feedback loop with the combination of ULPA filter just beneath the HEPA filter which is five orders of magnitude better than that of super cleanroom (ISO class 3). This result is obtained by controlling the number of particles coming out from the HEPA filter with the conjunction of the ULPA filter just beneath the HEPA filter. Other important parameters for obtaining the cleanliness of ISO class minus 2 are that the physically adsorbed particles coming out from the wall of the stainless steel is $2.3 \times 10^{-6} \mathrm{~m}^{-2} \mathrm{~s}^{-1}$ and the perfect air filtration through the filter of the LASAIR 310 particle counter. Finally, it can be concluded that the SCUSP with flat feedback loop would be a vital economical tool for the cutting-edge research and production platform such as nanotechnologies and biotechnologies.

\section{Acknowledgement}

The authors would like to thank Oshitari Lab., Inc., Bioflexe Inc., Synthemec Corp., Sysmex Corp., SONAC Inc., and Transtech. Inc. for filter and machine related aids for this study. This work is supported, in part, by grants from Hokkaido TLO, Sapporo City, Hokkaido Univ. R\&BP, Japan Science and Technology Agency (JST), Special
Education and Research Expenses from Post-Silicon Materials and Devices Research Alliance, and Nano-Macro Materials Devices and System Research Alliance.

\section{References}

1. Schicht, H. H, 1990. Contamination control-an indispensable factor in high-technology manufacturing tasks. J. of Aero. Science, 21, S719-S732.

2. Granneman, E. H. A, 1997. Trends in contamination control in IC production tools. Solid State Technology, 40, 225-232.

3. Whyte, W, 2001. Cleanroom Technology: Fundamentals of Design, Testing and Operation. John Wiley \& Sons, Chichester, UK.

4. Matts Ramstorp, 2000. Introduction to Contamination Control and Cleanroom Technology. WILEY- VCH Verlag $\mathrm{GmbH}$.

5. Havet, M. and F. Hennequin, 1999. Experimental characterization of the ambience in a food-processing clean room. J. Food Eng., 39, 329-335.

6. Yang, L. and C. E. Gan, 2007. Costing of Small Cleanroom. Building and Environment, 42, 743-751.

7. $\mathrm{Xu}, \mathrm{T}, 2008$. Characterization of minienvironment in a cleanroom: Assessing energy performance and its applications. Building and Environment, 43, 1545-1552.

8. Hu, S. C., Y. K. Chuah, and M. C. Yen, 2002. Design and evaluation of a minienvironment for semiconductor manufacture processes. Building and Environment, 37, 201208.

9. Ishibashi, A. and K. Kondo, 2004. Current-injection induced dislocation networks in II-VI laser diodes: bottom-up structures emerging in top-down system. Electron. Lett., 40, 1268-1269.

10. Ishibashi, A., H. Kaiju, Y. Yamagata, and N. Kawaguchi, 2005. Connected box-units-based compact highly clean environment for cross-disciplinary experiments platform. Electron. Lett., 41, 735-736

11. Kaiju, H., N. Kawaguchi, and A. Ishibashi, 2005. Study of very low airborne particle count in clean-unit system platform. Rev. Sci. Instrum., 76, 085111.

12. Dalilur Rahaman, Md., H. Kaiju, N. Kawaguchi, and A. Ishibashi, 2008. Ultra-High Cleanliness of ISO Class Minus 1 Measured in Triply Connected Clean-Unit System Platform. Jpn. J. App. Phys., 47, 5712-5716.

13. Ohmi, T, 1993. ULSI reliability through ultraclean processing. Proceedings of the IEEE, 81, 716-729. 